\title{
Store equity: teste do modelo teórico em uma rede de varejo
}

Helena Belintani Shigaki

Doutoranda em Administração com ênfase em Marketing pela Universidade Federal de Minas Gerais (UFMG) com período sanduíche na École des Hautes Études Commerciales de Montréal (HECMontréal), Mestre em Administração pela Pontifícia Universidade Católica de Minas Gerais

belintanihs@gmail.com

Antônio Carlos Rodrigues

Doutorando em Administração na Universidade Federal de Minas Gerais.Professor assistente da Universidade Federal de Juiz de Fora

tonimacr@gmail.com

Sâmara Borges Macedo

Doutoranda em Administração pela UFMG- Universidade Federal de Minas Gerais. Mestre em Administração na área de concentração Gestão Estratégica, Marketing e Inovação pela UFLA Universidade Federal de Lavras

samaraborgesmacedo@outlook.com

Caio Cesar Giannini Oliveira

Doutor e Mestre em Administração na PUC Minas. Coordena a Especialização em Comunicação Digital do IEC PUC Minas e coordenador do LabSG - laboratório de produção audiovisual (áudio, foto e vídeo) da PUC Minas.

caiocgo@gmail.com

\section{Editor Científico: José Edson Lara \\ Organização Comitê Científico}

Double Blind Review pelo SEER/OJS

Recebido em 16.01.2019

Aprovado em 09.09.2019 


\title{
Resumo
}

Objetivo do estudo: Testar o modelo teórico formativo de Store Equity em uma rede de varejo do ramo farmacêutico.

Metodologia/ abordagem: A pesquisa é descritiva e quantitativa, com 306 participantes. O trato dos dados foi conduzido por meio da Análise Fatorial Confirmatória com vistas a explicar a correlação e covariância entre as variáveis.

Originalidade/ relevância: O modelo, originalmente, é composto pelos antecedentes lealdade, lembrança, imagem e qualidade. A obtenção de um bom nível de Store Equity significa que os consumidores se lembrarão conscientemente da loja, mantendo uma imagem favorável e percebendo-a como uma organização de qualidade, permanecendo, então, leais a ela. A presente pesquisa visa contribuir para a validação do modelo, visto que das 54 citações ao modelo original, nenhum outro trabalho se propôs a apresentar tal validação.

Principais resultados: Os construtos do modelo atuam de maneira complementar, onde cada um influencia os demais e, juntos, compõem o valor que o consumidor atribui à loja.

Contribuições teóricas/ metodológicas: A teoria acerca dos construtos antecedentes do modelo, especialmente lealdade, foi revisitada e o questionário foi modificado com para suprir os problemas de mensuração anteriormente identificados. Entretanto, percebeu-se a baixa aderência da dimensão Imagem neste trabalho. Nesse sentido, entende-se que as investigações devem prosseguir e a realização de novas pesquisas no sentido de se chegar a um modelo mais consistente para a identificação do Store Equity é encorajada.

Palavras-chave: Lealdade, Lembrança, Imagem, Qualidade, Varejo.

\section{Store equity: test of the theoretical model in a retail sale}

\begin{abstract}
Objective of the study: This work aims to test Store Equity's theoretical formative model in a pharmaceutical retail chain.

Methodology/ approach: Methodologically, the research is descriptive and quantitative, with 306 respondents. The data treatment was conducted through Confirmatory Factor Analysis to explain the correlation and covariance between the variables.

Originality/ relevance: The model was originally built with the components loyalty, memory, image and quality. Achieving a good level of Store Equity means that consumers will consciously remember the store, maintain a favorable image, perceive it as a quality organization, and remain loyal to it. The present research aims to contribute to the validation of the model, since none of the 54 citations has successfully proposed to present such validation.
\end{abstract}


Main results: The procedures performed made it possible to conclude that the model constructs act in a complementary way, where each one influences the others and, together, make up the value that the consumer attributes to the store.

Theoretical/ Methodological contributions: The theory about the Store Equity model components, especially loyalty, was revisited and the questionnaire was modified to address previously identified measurement problems. However, we noticed the low adherence of the Image dimension in this work. In this sense, it is understood that investigations should continue and further research to arrive at a more consistent model for the identification of Store Equity is encouraged.

Keywords: Loyalty, Memory, Image, Quality, Retail.

\section{Store equity: teste del modelo teórico en una venta minorista}

\section{Resumen}

Objectivo del estudio: Este trabajo tiene como objetivo probar el modelo formativo teórico de Store Equity en una cadena minorista farmacéutica.

Metodologia/ enfoque: Metodológicamente, la investigación es descriptiva y cuantitativa, con 306 encuestados. El tratamiento de los datos se realizó a través del análisis factorial confirmatorio para explicar la correlación y la covarianza entre las variables.

Originalidad/ relevancia: El modelo fue construido originalmente con sus componentes son lealtad, memoria, imagen y calidad. Lograr un buen nivel de Store Equity significa que los consumidores recordarán conscientemente la tienda, mantendrán una imagen favorable, la percibirán como una organización de calidad y se mantendrán leales. La presente investigación tiene como objetivo contribuir a la validación del modelo, ya que ninguna de las 54 citas ha propuesto con éxito presentar dicha validación.

Resultados principales: Los procedimientos realizados permitieron concluir que las construcciones modelo actúan de manera complementaria, donde cada una influye sobre las demás y, juntas, conforman el valor que el consumidor atribuye a la tienda.

Contribuciones teóricas/ metodológicas: Se revisó la teoría sobre los componentes del modelo Store Equity, especialmente la lealtad, y se modificó el cuestionario para abordar problemas de medición previamente identificados. Sin embargo, notamos la baja adherencia de la dimensión Imagen en este trabajo. En este sentido, se entiende que las investigaciones deben continuar y se recomienda realizar más investigaciones para llegar a un modelo más coherente para la identificación de Store Equity.

Palabras clave: Lealtad, Recuerdo, Imagen, Calidad, Retail. 


\section{Introdução}

Compreender como o consumidor grava a imagem de uma loja na sua mente é um tópico que os pesquisadores têm se dedicado desde o fim da década de 1950, quando o conceito de imagem de loja foi cunhado por Martineu (1958). A partir desse, foram somados à literatura o valor da marca, ou Brand Equity, que pode ser analisado sob a ótica do consumidor, da empresa e financeira (Keller \& Lehmann, 2006). Hartman e Spiro (2005, p. 1113) desenvolveram a teoria em torno do conceito de Store Equity, considerando-o "o efeito diferencial do conhecimento da loja na resposta do consumidor frente às atividades de marketing dessa loja". Esse conceito foi analisado minuciosamente, de forma fragmentada e em conjunto neste artigo.

Como a imagem da loja é fixada na mente do consumidor, pesquisadores tem se preocupado em entender como se dá a associação entre o nome da loja, objetos, lugares e pessoas. Essa compreensão, também, é estudada a partir do conceito de Store Equity, com o intuito de compreender como os consumidores respondem ao marketing das lojas e qual tem sido seu efeito (Hartman \& Spiro, 2005). Partindo desses pressupostos, o modelo teórico de Story Equity é composto por quatro construtos antecedentes: Lealdade à loja, Lembrança da loja, Imagem da loja e, Qualidade percebida.

Dada a importância em se conhecer o consumidor e seu comportamento e, considerando-se a imagem da loja, o valor da marca, como os consumidores lidam com isso e qual o reflexo disso, o presente trabalho tem como objetivo testar o modelo proposto pelos autores Teixeira, Oliveira e Pires $(2006,2007)$ a partir da proposta teórica de Hartman e Spiro (2005), composto pelos quatro construtos do Store Equity.

Após a publicação desse estudo de Hartman e Spiro (2005), pouco mais de 200 trabalhos surgiram no decorrer dos anos e diversas aplicações foram realizadas em diferentes países, como por exemplo o varejo local sob o olhar do turista (Sharma, Chen, \& Luk, 2018), a lealdade no varejo (Gil-Saura et al., 2016), intenções comportamentais em um varejo de tecnologia (GilSaura, Molina, \& Berenguer-Contri, 2016), influência da imagem no consumo (Diallo, Burt, \& Sparks, 2015) e a maioria entrelaçando as teorias de Brand Equity com Store Equity, como o estudo de Sasmita e Suki (2015). Neste presente estudo, optou-se por manter a originalidade do trabalho desenvolvido por Hartman e Spiro em 2005 e, para tal, os trabalhos desenvolvidos por Teixeira, Oliveira e Pires $(2006,2007)$ mantém este princípio. 
A estrutura do trabalho, a começar por esta introdução, é a que segue: Fundamentação Teórica com um breve retorno à história e conceitos que circundam a teoria de Brand Equity, seguida pela do Store Equity e seus quatro construtos antecedentes e finalizada com a apresentação das questões utilizadas para teste do modelo. Em seguida, a metodologia com o percurso adotado, presando pela transparência e objetivando a validação externa por meio das possíveis replicações das constatações aqui presentes, por outros pesquisadores. Mais adiante, a análise dos dados com identificação das variáveis que compõem e fortalecem o modelo. Por fim, as considerações finais e as referências.

\section{Referencial teórico}

\subsection{Brand Equity}

A definição de marca passou a ser, então, voltada ao pensamento supracitado. Desde 2010, a American Marketing Association (AMA) a considera um termo, um nome, design, símbolo ou qualquer outro aspecto que que caracterize e identifique uma empresa fazendo com que a diferencie de outra. E, mais recentemente, como uma experiência do consumidor representada por uma coleção de imagens e ideias, que se referem a um símbolo, tal qual um nome, logotipo, slogan e design.

Relacionada a essa mudança no entendimento e conceituação de marca, têm-se o conceito de valor da marca, ou Brand Equity. O termo valor da marca é considerado tradução não só de Brand Equity, como também brand value, que, segundo Oliveira e Luce (2011), há trabalhos que fazem a distinção dos termos e outros que não o fazem. No entanto, a de se convir que a complexidade em se definir o termo valor reflete na multiplicidade de conceituações para valor da marca (Oliveira \& Luce, 2011; Nikhashemi et al., 2016).

O despertar do uso dessa terminologia se deu no início da década de 80 (Aaker, 1996; Yoo \& Donthu, 2001; Keller \& Lehmann, 2006; Christodoulides \& Chernatony, 2010) e pode ser compreendida e definida sob três perspectivas: sob a ótica financeira, da empresa e a do consumidor (Keller \& Lehmann, 2006).

Sob a ótica financeira, compreende-se o termo como o valor (preço) que se pode obter com a venda da marca, ou seja, o valor proporcionado pelo nome da marca, compreendendo-a semelhante a um equipamento que pode ser comprado e vendido. Sob a ótica da empresa, uma 
marca consolidada proporciona maior proteção em relação à concorrência, maiores margens e melhor resposta em relação a aumento de preços (Keller \& Lehmann, 2006). Sob a ótica do consumidor, o foco é dado à preferência que o mesmo atribui ao nome da marca, ou seja, a atração por um produto específico, dada a marca que o mesmo carrega e não seus atributos especificamente (Christodoulides \& Chernatony, 2010).

Alguns pontos são levantados como cruciais no contexto do Brand Equity: a publicidade (Yoo \& Donthu, 2001; Keller \& Lehmann, 2006), a lealdade - podendo estabelecer uma ligação pessoal com a marca, implicando em compromisso com a mesma (Sasmita e Suki, 2015) e, o envolvimento com a marca (Rao, 2012). A idade da marca, ou sua longevidade, é vista como fonte de valor, a medida que pode proporcionar produtos com maior qualidade refletindo em lealdade, notoriedade e até mesmo em percepção ética (Iglesias et al., 2019). Outra fonte de valor defendida pelos mesmos autores é a ordem de entrada no mercado, que consequentemente afeta o Posicionamento da Marca, fator relevante para o construto de Brand Equity (Rossoni \& Guarido Filho, 2015).

Segundo Oliveira e Luce (2011), existem vários modelos para estimar o Brand Equity, no entanto, nenhum possui plena aceitação. Aaker (1996) relaciona o conceito de Brand Equity à geração de valor proporcionada pela marca para os consumidores e empresas. Para os consumidores medida que, atrelada à qualidade percebida, gera maior satisfação e confiança, e para as empresas, possibilita maior margem de preço e vantagem competitiva (Aaker, 1996). Levando em consideração o exposto, o autor, assim como Keller (2001), identificou cinco dimensões relacionadas à Brand Equity: lealdade, notoriedade da marca, qualidade percebida, associações à marca e outros ativos da empresa, sendo que, na visão de Aaker (1996), a lealdade aparece como dimensão central.

Baseando-se nos dois autores ora mencionados, Yoo e Donthu (2001), relacionaram três dimensões ao Brand Equity: lealdade à marca, notoriedade da marca e qualidade percebida. A pesquisa desenvolvida pelos autores se deu por meio do desenvolvimento e validação de uma escala de medição de Brand Equity sob a ótica do consumidor. Os autores consideraram inicialmente quatro dimensões (lealdade, notoriedade da marca, qualidade percebida, associações à marca), no entanto, ao entenderem a dimensão associações à marca indissociável das outras, tomaram como dimensões relacionadas à Brand Equity as três primeiras. 
A subseção seguinte visa expor a definição e os elementos que compõem o Store Equity, termo cunhado por Hartman e Spiro (2005), com origem em estudos sobre Brand Equity e mais especificamente Imagem da loja.

\subsection{Store Equity}

O termo Store Equity foi apresentado inicialmente no trabalho de Hartman e Spiro (2005) ao partirem da premissa da teoria de Store Image de Martineau (1958) em que uma loja passa a ser definida a partir da mente dos consumidores ou visitantes (shoppers), de suas qualidades funcionais e de seus atributos psicológicos. Partindo desse horizonte, Store Equity é definido como "o efeito diferencial do conhecimento da loja na resposta do consumidor frente às atividades de marketing dessa loja" (Hartman \& Spiro, 2005, p. 1113). O esclarecimento de cada termo desse conceito foi descrito a seguir.

O efeito diferencial, primeiro componente da definição ora mencionada, reflete a capacidade subjetiva e objetiva do consumidor ao comparar objetos, lugares e pessoas com seus similares, embora distintos (Hartman \& Spiro, 2005). As autoras lembram ainda que este componente se refere à comparação pura e simples de uma rede de lojas e outras que por ventura o consumidor conheça e consiga estabelecer associação ou relação. O conhecimento da loja, segundo componente da definição de Store Equity, é conceituado pelas mesmas autoras (2005) como o nome da loja fixado na memória do consumidor, juntamente com uma variedade de associações que são desencadeadas. Esse conceito também é referenciado pelo store image. A resposta dos consumidores, terceiro e último componente da definição, refere-se às respostas dos consumidores frente às atividades de marketing, definidas pelas respostas desses à suas preferências e seus comportamentos, frente às ações de outras lojas (Hartman \& Spiro, 2005).

O artigo seminal de 2005, de Hartman e Spiro, foi citado 286 vezes no Google Scholar e 87 na Web of Science - segundo dados da busca realizada em outubro de 2018 -, e dessas, foi possível constatar que a maioria dos autores ainda trabalha o conceito de store image separadamente e, por vezes, os construtos de qualidade, lealdade, mix de produtos e estudos comparativos de Brand Equity entre lojas, medindo serviços, dinâmicas e motivações do consumidor. Outro achado interessante foi o uso do termo retail equity, que sugere uma replicação similar ao conceito de Brand Equity (Pappu \& Quester, 2006) sugerindo uma abordagem voltada para o consumidor e outra para aferição dos índices do varejo, 
compreendidos por lembrança, associação, qualidade, lealdade e valor percebido (Arnett, Laverie, \& Meiers, 2003). Nesse sentido, pode-se afirmar que este novo conceito se assemelha à teoria aqui estudada e que os autores optaram por manter o termo tal qual o original: Store Equity.

São quatro os antecedentes que compõem o modelo teórico formativo e fundamentam o conceito de Store Equity: lealdade à loja, lembrança da loja, imagem da loja e, qualidade percebida, como pode ser observado na Figura 1 e nas descrições presentes nas subseções a seguir.

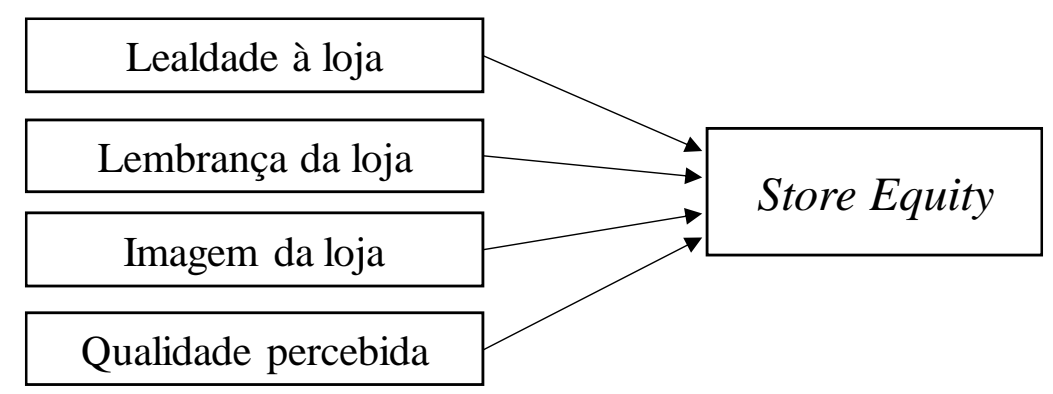

Figura 1. Modelo conceitual do Store Equity

Fonte: Adaptado de Teixeira, Oliveira e Pires (2006; 2007)

\subsubsection{Lealdade à loja}

Definir lealdade não é uma tarefa fácil. Há diversas definições e contradições na literatura, que muitas vezes se confundem com os conceitos de satisfação e fidelidade, por exemplo. Dick e Basu (1994) classificaram a lealdade em componentes atitudinais e comportamentais, resultando em quatro categorias: (a) lealdade verdadeira: combinação de atitude e comportamentos fortes, ou seja, além da repetição de compras, o consumidor é comprometido; (b) lealdade latente: combinação de comportamento fraco com fortes atitudes, indicando baixa conduta de recompra, como por exemplo, preço inacessível; (c) lealdade falsa: combinação de comportamento forte com atitude baixa, ou seja, não há comprometimento, mas existe uma recompra por fatores ocasionais, por exemplo: baixo preço e, (d) falta de lealdade, ou seja, quando não há comportamento de recompra.

Esse conceito pode ser considerado uma evolução do pensamento de Brown (1952) ao considerar que a lealdade pode ser dividida. Segundo ele, a lealdade não dividida (undivided loyalty) ocorre quando um cliente compra produtos ou serviços somente de uma loja, exemplo: AAAAAA; a lealdade dividida (divided loyalty) ocorre quando um cliente compra 
alternadamente nas lojas A e B, exemplo: $\mathrm{ABABAB}$ e; existe também a falta de lealdade (irregular sequences), ou seja, quando o cliente não é leal e compra em qualquer loja, exemplo; ABBACDB.

A lealdade pode ser considerada ainda como intenção de repetição de compra, que influencia na opção de mudança na escolha do consumidor (Das, 2016; Ahrholdt, Gudergan, \& Ringle, 2019); ou mesmo como o comprometimento do cliente com a empresa, que é manifestado diante das repetidas compras (Song, Wang, Han, 2019). Outros afirmam, ainda, que para se ter lealdade, a satisfação é um pré-requisito para a retenção do cliente (Oliver, 1999). Oliver (1999), partindo do método de divisão de Brown (1952) entende que a lealdade pode ser dividida em cognitiva (informações, avaliações, atributos), afetiva (gosto, satisfação, busca), conativa (comprometimento, experiências) e de ação (lealdade à inércia).

\subsubsection{Lembrança da loja}

A lembrança da loja, por vezes, é confundida com o conhecimento ou associação à loja. Isso porque o conceito foi construído a partir da perspectiva da consciência da marca, que é composta pelo reconhecimento e pela lembrança. E, segundo Keller (2001), o reconhecimento é a habilidade que o consumidor tem em (re)lembrar de uma marca mediante exposição a categoria a qual ela pertence.

Para Hartman e Spiro (2005), a lembrança da loja é considerada como o reconhecimento de uma loja frente às outras do mesmo ramo de atuação. A interpretação de Teixeira, Oliveira e Pires $(2006 ; 2007)$ é que o consumidor consiga identificar uma loja sob diferentes condições, por exemplo: exposições, apresentação do nome, ou em comparação com outra loja da mesma categoria de varejo.

A lembrança pode ser medida de três diferentes maneiras, segundo Romaniuk et al. (2004): (a) top of mind, ou seja, a primeira marca que vem à mente do consumidor, (b) espontâneo, ou seja, quando o consumidor se lembra da marca de forma automática e, (c) auxiliado, ou seja, quando o consumidor se lembra da marca mediante o fornecimento de informações. De acordo com o mesmo autor, a lembrança é principalmente utilizada para medir o valor de uma loja e a sua eficácia de comercialização. 


\subsubsection{Imagem da loja}

Hartman e Spiro (2005) partiram da teoria de imagem da loja de Martineau (1958) para definição do conceito de Store Equity. Para elas, a imagem pode ser medida frente à atmosfera, merchandising, conveniência, funcionários, entre outros. Já segundo a interpretação de Teixeira, Oliveira e Pires (2006; 2007), é um conjunto de percepções de caráter funcional e psicológico acerca de uma determinada loja na mente do consumidor.

A imagem da loja também pode ser definida como a percepção dos consumidores, formada pelos componentes cognitivos, afetivos e conativos, em relação a uma loja (Song, Wang, Han, 2019), além de oferecer vantagem competitiva diante dos demais concorrentes.

São diversos os atributos que formam o conceito de imagem, e dentre eles destacam-se: preço, qualidade, mix de produtos e serviços, pessoas, conveniência, serviços, promoções, publicidade, atmosfera, fator institucional, preferência do consumidor, layout, impressão geral da loja, relação custo-benefício, reputação, música, design, entre outros (Giraldi, Spinelli, \& Merlo, 2003; Thang \& Tan, 2003).

Quando uma loja se preocupa em manter uma boa imagem frente ao consumidor, ela automaticamente produz um impacto positivo no cliente, sendo possível a utilização de preços premiuns e servindo de base para o processo de fidelização (Martenson, 2007).

\subsubsection{Qualidade percebida}

A qualidade é definida como um benefício percebido mediante julgamento do consumidor, que pode vir a gerar uma satisfação (Sharma, Chen, \& Luk, 2018), considerando aqui e em todos os outros construtos do modelo Store Equity a subjetividade, a experiência anterior desse e as possíveis e recorrentes comparações (Fornell et al., 1996).

A qualidade pode, ainda, ser considerada uma das principais razões de compra em determinados países (Sharma, Chen, \& Luk, 2018). Consumidores que estão em países que já possuem esse fator como prioridade na compra e venda de produtos e serviços, certamente priorizam outros fatores como preço, interação e experiência de compra (Sharma, Chen, \& Luk, 2018).

Mangold e Faulds (1993) identificaram, por meio de uma pesquisa empírica no varejo, seis níveis que influenciam e são influenciados pela qualidade: interação pessoal dos 
funcionários com consumidores, políticas de marketing, arranjo físico da loja, promessas de vendas, soluções de problemas e conveniência. A qualidade percebida, tanto do serviço quanto do produto, é avaliada pelo consumidor a todo instante e a loja deve estar atenta em manter uma organização a fim de atendê-lo com excelência, causando uma boa impressão e impactando na satisfação do consumidor e demais stakeholders.

\section{Metodologia}

Para atingir o objetivo do estudo, o de teste do modelo teórico de Hartman e Spiro (2005) a partir das pesquisas de Teixeira, Oliveira e Pires (2006; 2007), realizou-se uma pesquisa com abordagem descritiva e do tipo quantitativa. A pesquisa descritiva foi escolhida por descrever o fenômeno do comportamento do consumidor e suas atitudes com base nos dados primários coletados, além de auxiliar na previsão de questões específicas, que é o caso do Store Equity. Por quantitativa, entende-se pela escolha da amostra e pela aplicação de um questionário estruturado e auto-administrado a 306 consumidores de uma loja em uma rede farmacêutica nacional. Considerando a recomendação de 5 a 10 casos por parâmetro (Hair Jr. et al., 2009), o tamanho da amostra é considerado satisfatório.

A partir da literatura existente e de trabalhos anteriores acerca do tema, foram elaboradas 15 questões sobre lealdade, lembrança, qualidade e imagem da loja, que podem ser observadas na Figura 2, a seguir.

\begin{tabular}{|c|c|c|c|}
\hline LEALDADE & LEMBRANÇA & QUALIDADE & IMAGEM \\
\hline $\begin{array}{c}\text { L01 }- \text { Sempre que me } \\
\text { pedem referências sobre } \\
\text { uma loja do ramo, indico } \\
\text { a Loja X }\end{array}$ & $\begin{array}{c}\text { A01 - Quando penso em } \\
\text { uma loja do ramo, a Loja } \\
\text { X é a primeira que me } \\
\text { vem à mente }\end{array}$ & $\begin{array}{c}\text { Q01 - Confio na } \\
\text { qualidade dos produtos e } \\
\text { nos serviços oferecidos } \\
\text { pela Loja X }\end{array}$ & $\begin{array}{c}\underline{\mathbf{I 0 1}} \text { - A localização } \\
\text { desta unidade da Loja X } \\
\text { atende minha } \\
\text { expectativa }\end{array}$ \\
\hline $\begin{array}{l}\text { L02 - Mais da metade } \\
\text { de minhas compras de } \\
\text { produtos/serviços de } \\
\text { drogaria e conveniência } \\
\text { são feitas na Loja X }\end{array}$ & $\begin{array}{l}\underline{\mathbf{A 0 2}} \text { - Sou capaz de } \\
\text { reconhecer a loja da Loja } \\
\text { X dentre outras do ramo }\end{array}$ & $\begin{array}{l}\text { Q02 - Sempre fui bem } \\
\text { atendido (a) na Loja X }\end{array}$ & $\begin{array}{l}\text { I02 }- \text { O ambiente físico } \\
\text { da Loja X é agradável }\end{array}$ \\
\hline $\begin{array}{l}\text { L03 - Considero a Loja } \\
\text { X como a minha } \\
\text { primeira escolha para as } \\
\text { compras de } \\
\text { produtos/serviços de } \\
\text { drogaria e conveniência }\end{array}$ & $\begin{array}{c}\underline{\mathbf{A 0 3}}-\text { Eu tenho uma } \\
\text { opinião formada sobre a } \\
\text { Loja X }\end{array}$ & $\begin{array}{c}\text { Q03 - Os produtos / } \\
\text { serviços de drogaria e } \\
\text { conveniência oferecidos } \\
\text { pela Loja X satisfazem } \\
\text { todas as minhas } \\
\text { necessidades }\end{array}$ & $\begin{array}{c}\underline{\mathbf{I 0 3}} \text { - Os preços das } \\
\text { mercadorias / serviços } \\
\text { encontrados na Loja X } \\
\text { estão de acordo com o } \\
\text { que estou disposto a } \\
\text { pagar }\end{array}$ \\
\hline
\end{tabular}




\begin{tabular}{cc}
\hline $\begin{array}{c}\mathbf{\text { O04}} \text { - Algumas } \\
\text { características da Loja X } \\
\text { vêm à minha mente } \\
\text { rapidamente }\end{array}$ & $\begin{array}{c}\mathbf{\text { O44 }}-\text { A Loja X } \\
\text { apresenta variedade } \\
\text { adequada de produtos / } \\
\text { serviços }\end{array}$ \\
$\begin{array}{c}\underline{\mathbf{I 0 5}}-\text { Eu consigo } \\
\text { identificar a Loja X pela } \\
\text { sua propaganda }\end{array}$ \\
\hline
\end{tabular}

Figura 2. Indicadores do modelo de Store Equity

Fonte: Elaborado pelos autores a partir de Teixeira, Oliveira e Pires (2006; 2007)

Anteriormente a esse estudo, foram realizados seis trabalhos com o mesmo intuito, sendo os primeiros o de Teixeira, Oliveira e Pires $(2006$; 2007), seguidos por quatro dissertações. A primeira foi a de Muniz (2009), com 30 questões diretamente relacionadas ao modelo, cujos resultados apontaram "deficiências no ajuste do modelo e ausência da validade discriminante do construto Qualidade” (p. 92). A seguinte, do Miranda (2009), utilizou o mesmo questionário do trabalho anterior e indicou que o construto Lembrança não apresentou uma relação positiva e significativa com o Store Equity e, com o construto imagem apresentou elevada correlação. No ano seguinte, os trabalhos de Drudi Jr (2010) e Oliveira (2010) fizeram uso de um questionário reduzido com 24 perguntas com exclusão e modificação dessas frente aos utilizados anteriormente. Como conclusão, os autores indicaram deficiências no ajuste do modelo principalmente no construto Lealdade.

Diante disso, o questionário aqui utilizado foi elaborado a partir dos últimos trabalhos empíricos realizados por Drudi Jr. (2010) e Oliveira (2010), que por sua vez se originaram a partir dos estudos de Teixeira, Oliveira e Pires (2006; 2007), Miranda (2009) e Munhoz (2009). Além disso, a teoria foi revisitava, tendo em vista que os trabalhos anteriores não foram completamente validados e apresentaram problemas de mensuração de algumas escalas. O novo questionário passou por uma avaliação de face com especialistas no assunto, além de um préteste para adequação final das perguntas.

Os questionários foram disponibilizados via Google Form e encaminhados via e-mail aos contatos dos pesquisadores do presente projeto. Foi utilizada a modalidade bola de neve, na qual um respondente indica outro respondente em caráter sucessivo e dois incentivos em vale compras de $\mathrm{R} \$ 100,00$ cada ao participante que mais indicou respondentes e um sorteio incluindo todos os participantes da pesquisa, no qual todos tiveram a mesma chance de concorrer ao prêmio. Os entrevistados foram selecionados por meio da técnica de amostragem não probabilística, por julgamento, tendo como pré-requisito ser consumidor de pelo menos uma das lojas de uma rede de varejo farmacêutica nacional. A escolha de uma única rede foi 
necessária diante da necessidade de padrão da loja, incluindo layout, preço, qualidade, mix de produtos e serviços, pessoas, conveniência, serviços, promoções, publicidade, atmosfera, fator institucional, preferência do consumidor, layout, impressão geral da loja, relação custobenefício, reputação, música, design, entre outros. A mesma pesquisa poderia ser feita, por exemplo, com uma única loja ou uma franquia (Hartman \& Spiro, 2005). Os resultados relativos à caracterização sócio-demográfica dos pesquisados foram segmentados por gênero, com as seguintes variáveis: estado civil, faixa etária e renda familiar.

Os dados ausentes (missing data) são informações não disponível a respeito de uma observação (ou caso) sobre a qual outras informações estão disponíveis (Hair Jr. et al., 2009). Não houve dados ausentes porque o instrumento de coleta de dados foi elaborado com respostas obrigatórias para todas questões relevantes. O questionário só era finalizado após o preenchimento de todas as respostas. Além disso, não foram identificados outliers nos dados referentes a desta pesquisa, estes não foram identificados. Por se tratar de uma análise multivariada, houve a necessidade de verificação do atendimento aos pressupostos de normalidade, linearidade, homocedasticidade, multicolinearidade e singularidade (Hair Jr. et al., 2009; Tabachinick \& Fidell, 2007).

Em resumo, este estudo tem como objetivo explicar a correlação ou covariância, entre um conjunto de variáveis, em termos de um número limitado de variáveis não observáveis, optou-se pela utilização de técnicas de Análise Fatorial (AF). Existem dois tipos de AF, que devem se adequar aos objetivos de cada pesquisa, cuja finalidade da sua aplicabilidade aconteça de forma exploratória e confirmatória (Tabachinick \& Fidell, 2007).

A Análise Fatorial Exploratória (AFE) é utilizada em estágios embrionários de pesquisa, como o intuito de explorar os dados, ou seja, a relação entre um conjunto de variáveis, em busca de padrões de correlação. A Análise Fatorial Confirmatória (AFC), por sua vez, é utilizada para testar hipóteses. O pesquisador suportado por alguma teoria testa o grau em que determinadas variáveis são representativas de uma dimensão ou conceito. Tendo em vista que o fenômeno foi discutido em trabalhos anteriores e que buscamos testar inter-relações para uma determinada amostra ou contexto de uma estrutura fatorial já conhecida na literatura, optou-se pela utilização do método AFC para demonstração dos resultados.

A AFC foi realizada utilizando o software AMOS versão 16 para análise estatística das variáveis latentes. A qualidade do ajustamento mensura a correspondência da matriz de dados 
de entrada com aquela prevista para o modelo proposto. $\mathrm{Na}$ aplicação desenvolvida, foram utilizadas as estatísticas de bondade de ajustes, nomeadamente: o qui-quadrado $\left(\chi^{2}\right)$, o Índice de Ajuste Comparativo (CFI), o Índice de Ajuste não Normalizado (NNFI), o Índice de Bondade de Ajuste Ajustado (AGFI), o Índice de Bondade de Ajuste (GFI) e o Erro Quadrático Médio Aproximado (RMSEA) (Kline, 2011).

\section{Apresentação e discussão dos resultados}

Para atender ao objetivo do trabalho, realizou-se a AFC e os resultados são apresentados nesta seção. A caracterização dos respondentes é a que segue: $52 \%$ são do sexo feminino e $48 \%$ do masculino. A maioria possui idade que varia de 20 a 31 anos (54\%), ensino superior completo (89\%) e com renda familiar superior a $\mathrm{R} \$ 4500,00(50 \%)$.

Testou-se a representatividade do modelo conceitual por meio Análise Fatorial Confirmatória (AFC) afim de verificar a amplitude com que a variáveis observadas selecionadas eram geradas pelos fatores correlacionados. Neste sentido, o foco centraliza-se nos coeficientes de regressão das variáveis observadas sobre os fatores. A avaliação do modelo de mensuração foi feita por meio dos índices de ajustes, da validade convergente e da validade discriminante.

Além da qualidade do ajustamento é necessário avaliar a confiabilidade e a validade de medição. A confiabilidade diz respeito ao atributo de consistência e reprodução da medida. Nesse sentido, uma medida de confiabilidade adequada para AFC é a confiabilidade composta (Fornell \& Larcker, 1981). A validade é o atributo da escala de medida que verifica se esta mede o construto que se pretende avaliar. Ou seja, a validade convergente é estabelecida quando os itens que são reflexos de um fator saturado e o comportamento destes itens é explicado, essencialmente, por este fator. Além disso, a validade discriminante verifica se os itens que refletem um fator não são relacionados com outros fatores (Hair Jr. et al., 2009; Kline, 2011).

A AFC foi realizada junto ao software estatístico AMOS versão 16. Para tanto, análises exploratórias iniciais foram realizadas para verificar a adequação dos dados aos pressupostos da AFC frente a análise de equações estruturais (Kline, 2011). A distribuição das pontuações dos 306 respondentes em cada um dos itens do modelo é apresentada na Tabela 1. Não foram 
observados problemas relativos à multicolinearidade, singularidade e casos extremos univariados e multivariados influentes (Tabachinick \& Fidell, 2007; Kline, 2011).

Tabela 1

Estatística descritivas dos itens

\begin{tabular}{|l|l|l|l|l|l|l|}
\hline Item & Média & Desvio Padrão & \multicolumn{2}{|l|}{ Assimetria } & \multicolumn{2}{|c|}{ Curtose } \\
\hline Leal_1 & 6,89 & 2,638 &,- 728 &, 139 &,- 030 &, 278 \\
\hline Leal_2 & 6,30 & 3,245 &,- 442 &, 139 & $-1,048$ &, 278 \\
\hline Leal_3 & 6,46 & 3,099 &,- 577 &, 139 &,- 749 &, 278 \\
\hline Lemb_1 & 7,97 & 2,637 & $-1,349$ &, 139 & 1,051 &, 278 \\
\hline Lemb_2 & 8,96 & 1,758 & $-1,964$ &, 139 & 3,714 &, 278 \\
\hline Lemb_3 & 8,26 & 2,320 & $-1,435$ &, 139 & 1,525 &, 278 \\
\hline Lemb_4 & 7,99 & 2,224 & $-1,077$ &, 139 &, 813 &, 278 \\
\hline Qual_1 & 8,73 & 1,599 & $-1,289$ &, 139 & 1,216 &, 278 \\
\hline Qual_2 & 7,84 & 2,222 & $-1,229$ &, 139 & 1,391 &, 278 \\
\hline Qual_3 & 7,35 & 2,257 &,- 794 &, 139 &, 318 &, 278 \\
\hline Imag_1 & 8,46 & 2,289 & $-1,831$ &, 139 & 3,103 &, 278 \\
\hline Imag_2 & 8,47 & 1,834 & $-1,518$ &, 139 & 2,571 &, 278 \\
\hline Imag_3 & 5,78 & 2,579 &,- 400 &, 139 &,- 421 &, 278 \\
\hline Imag_4 & 8,24 & 1,677 & $-1,074$ &, 139 & 1,749 &, 278 \\
\hline Imag_5 & 8,57 & 2,017 & $-1,660$ &, 139 & 2,733 &, 278 \\
& & & & & & \\
\hline
\end{tabular}

Nenhuma das variáveis apresentou valores de assimetria e curtose indicadores de violações graves à distribuição normal (Hair Jr. et al., 2009). A qualidade global do ajustamento do modelo fatorial foi realizada de acordo com os índices $\chi 2 /$ gl, CFI, GFI, AGFI e RMSEA.

A AFC do modelo para 4 fatores (lealdade, lembrança, imagem e qualidade) revelou um ajustamento ruim à matriz de variância-covariância para os 15 itens $(\chi 2 / \mathrm{gl}=4,088 ; \mathrm{CFI}=0,853$; $\mathrm{GFI}=0,855, \mathrm{AGFI}=0,793, \mathrm{RMSEA}=0,101)$. $\mathrm{O}$ ajuste do modelo foi produzido a partir dos índices de modificações sugeridos pelo software AMOS e fundamentados em considerações teóricas.

Feita a remoção dos itens Lemb_1, Imag_2 e Imag_5 foi possível alcançar uma boa qualidade de ajustamento $(\chi 2 / \mathrm{gl}=3,666 ; \mathrm{CFI}=0,908 ; \mathrm{GFI}=0,908, \mathrm{AGFI}=0,851, \mathrm{RMSEA}=$ 0,093). O novo modelo é então constituído por 4 fatores com 3 itens cada, como exposto na Figura 3. 


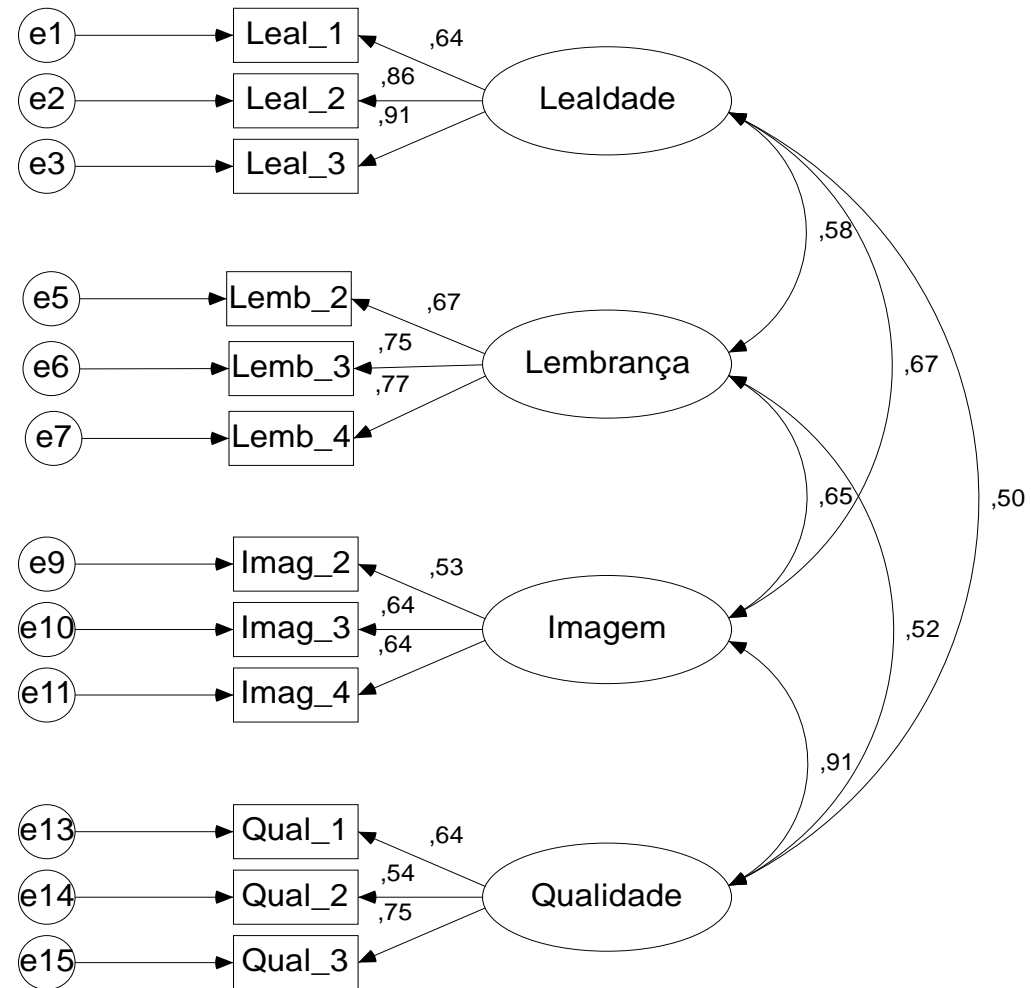

\section{Figura 3. Modelo}

Fonte: Elaborada pelos autores

Dado a limitação no teste de alfa de Cronbach, responsável pela avaliação da influência individual dos construtos, foi preferencialmente utilizada a confiabilidade composta dos fatores que considera a influência dos demais construtos simultaneamente (Fornell \& Larcker, 1981). Este indicador mostrou-se adequado, com valores de 0,849 para Lealdade e de 0,777 para Lembrança e próximo do adequado, com valores de 0,632 para Imagem e de 0,681 para Qualidade.

A validação do modelo é o último requisito para dar suporte a decisão de aceitar ou rejeitar, nas quais são analisadas a confiabilidade e a validade dos construtos. A validade convergente é demonstrada quando o construto em estudo se correlaciona, positiva e significativamente, com outros construtos teoricamente paralelos e quando os itens que constituem o construto apresentam correlações positivas e elevadas entre si. Seguindo Fornell e Larcker (1981), a variância extraída média (AVE), proposta como um indicador desta validade, foi utilizada no modelo revelando valores adequados para os construtos, sendo de 0,657 para Lealdade, de 0,538 para Lembrança, de 0,366 para Imagem, de 0,420 para Qualidade conforme ilustrado na Tabela 1. 
A análise da validade discriminante pode ser realizada comparando-se as AVE para cada construto com o quadrado da correlação entre os construtos cuja validade discriminante se pretende analisar. Assim utilizou-se os valores calculados para avaliar se os itens que refletem o construto não estão correlacionados com outros construtos, conforme indica a Tabela 2.

\section{Tabela 2}

Validade Convergente, Confiabilidade e Validade Discriminante (Critério de FornellLarcker)

\begin{tabular}{|l|c|c|c|c|}
\hline & Lealdade & Lembrança & Imagem & Qualidade \\
\hline Leal_3 & 0,909 & & & \\
\hline Leal_2 & 0,861 & & & \\
\hline Leal_1 & 0,635 & & & \\
\hline Lemb_4 & & 0,770 & & \\
\hline Lemb_3 & & 0,754 & & \\
\hline Lemb_2 & & 0,672 & & \\
\hline Imag_4 & & & 0,640 & \\
\hline Imag_3 & & & 0,642 & \\
\hline Imag_2 & & & 0,526 & \\
\hline Qual_3 & & & & 0,749 \\
\hline Qual_2 & & & & 0,541 \\
\hline Qual_1 & & & 0,638 \\
\hline Variância Média Extraída (AVE) & 0,657 & 0,538 & 0,366 & 0,420 \\
\hline Confiabilidade Composta (CC) & 0,849 & 0,777 & 0,632 & 0,681 \\
\hline Critério de Fornell-Larcker* & Lealdade & Lembrança & Imagem & Qualidade \\
\hline Lealdade & 0,657 & & & \\
\hline Lembrança & 0,333 & 0,538 & & \\
\hline Imagem & 0,449 & 0,420 & 0,366 & \\
\hline Qualidade & 0,250 & 0,271 & 0,819 & 0,420 \\
\hline
\end{tabular}

Nota: Números na diagonal principal são as AVEs e números fora da diagonal são os valores correlações entre os constructos do modelo elevados ao quadrado.

Verifica-se que todas as AVE são superiores ou iguais ao quadrado da correlação entre os construtos, sendo única exceção a correlação entre os construtos Qualidade e Imagem, cujo valor não constitui uma diferença de comparação relevante, de modo que, no geral, fica estabelecida a validade discriminante do modelo. De fato, o procedimento executado permite que seja percebido que a Imagem, a Qualidade, a Lembrança e a Lealdade atuam de maneira complementar; cada um destes elementos influencia os demais e, juntos, compõem o valor que o consumidor atribui à loja. A proximidade dos valores de cada um destes elementos reforça esta interdependência na construção do Store Equity. A qualidade percebida influencia a 
imagem formada acerca da loja e, como consequência a lembrança que se constrói acerca do estabelecimento e a propensão a repetir a experiência de compra (lealdade). No entanto, os dados obtidos no procedimento executado para esta investigação indicaram que tanto na AFE quanto na AFC, a dimensão Imagem não apresenta aderência como o modelo.

\section{Considerações finais}

Este trabalho pretendeu contribuir para o teste do modelo proposto pelos autores Teixeira, Oliveira e Pires (2006, 2007) a partir da proposta teórica de Hartman e Spiro (2005), composto pelos construtos antecedentes lealdade à loja, qualidade percebida, imagem e lembrança da loja. O modelo dos autores foi aqui escolhido para ser testado diante das várias tentativas desses e de outros autores de se chegar a um modelo de Store Equity. Ademais, das 54 citações à Hartman e Spiro (2005), nacionais e internacionais, nenhum outro autor se propôs a apresentar um modelo teórico que fosse factível de validação.

Tendo em vista os achados encontrados e as deficiências apontadas por outros autores discutidas anteriormente, a teoria acerca dos construtos antecedentes do modelo, especialmente lealdade, foi revisitada e o questionário foi modificado com a finalidade de suprir os problemas de mensuração anteriormente identificados, passando por uma avaliação de face e um pré-teste. No entanto, mesmo diante das modificações, pode-se afirmar que o estudo não cessa aqui e que novas pesquisas devem ser realizadas para que se tenha validação e robustez necessárias para a confirmação do modelo proposto de Store Equity.

Apesar das limitações apresentadas, a contribuição do trabalho se dá principalmente na busca pelo teste do modelo proposto. Embora tenha-se percebido a baixa aderência da dimensão Imagem obtida neste trabalho, entende-se que as investigações devem prosseguir e a realização de novas investigações no sentido de se chegar a um modelo mais consistente para a identificação do Store Equity é encorajada.

No contexto atual em que se percebe o acirramento da concorrência entre as empresas que trabalham com atendimento direto ao consumidor, é possível entender que estes diferenciais agregados aos produtos oferecidos representam peso na construção da experiência de compra. Este aspecto - a experiência - tem se mostrado preponderante para a valoração e, consequentemente, a fidelidade do consumidor (Larán \& Espinoza, 2004). Compreendendo que 
estes elementos se correlacionam, torna-se ainda mais importante evoluir a investigação acerca da validação do modelo Store Equity.

A obtenção de um bom nível de Store Equity significa, portanto, que os consumidores possuem se lembrarão conscientemente da loja, mantendo uma imagem favorável e percebendo-a como uma organização de qualidade, permanecendo, então, leais a ela. No atual mercado competitivo, Das (2016) alerta que o resultado final dessa função, a lealdade, reflete em um relacionamento benéfico de longo prazo entre consumidor e varejo.

\section{Referências}

Aaker, D. A. (1996). Measuring Brand Equity across products and markets. California Management Review, 3(3), 102-120.

Ahrholdt, D.C., Gudergan, S., \& Ringle, C.M. (2019). Enhancing loyalty: When improving consumer satisfaction and delight matters. Journal of business research, 94, 18-27.

AMA. (2018). American Marketing Association's Dictionary. Recuperado de https://www.ama.org/resources/Pages/Dictionary.aspx?dLetter=B.

Arnett, D. B., Laverie, D. A., \& Meiers, A. (2003). Developing parsimonious Retailer Equity indexes using partial least squares analysis: a method and applications. Journal of retailing, 79 (3), 161-170.

Brown, G. H. (1952). Brand Loyalty: Fact or Fiction. Advertising Age, 23, 53-55.

Christodoulides, G., \& Chernatony, L. (2010). Consumer-based brand equity conceptualization and measurement: A literature review. International journal of research in marketing, 52 (1), 43-66.

Das, G. (2016). Antecedents and consequences of trust: an e-tail branding perspective. International Journal of Retail \& Distribution Management, 44 (7), 713-730.

Diallo, M.F., Burt, S., \& Sparks, L. (2015). The influence of image and consumer factors on store brand choice in the Brazilian market: Evidence from two retail chains. European Business Review, 27 (5), 495-512.

Dick, A. S., \& Basu, K. (1994). Customer loyalty: toward an integrated conceptual framework. Journal of the academy of marketing science, 22 (2), 99-113.

Drudi Jr., J. (2010). Aplicabilidade do modelo Store Equity: um estudo em uma panificadora na cidade de São Paulo. Dissertação de Mestrado, Pontifícia Universidade Católica de Minas Gerais, Belo Horizonte, MG, Brasil.

Fornell, C., Johnson, M. D., Anderson, E. W., Cha, J., \& Bryant, B. E. (1996). The American Customer Satisfaction Index: Nature, Purpose, and Findings. Journal of Marketing, 60 (4), 7-18.

Fornell, C., \& Larcker, D. (1981). Evaluating structural equation models with unobservable variables and measurement error. Journal of Marketing Research, 18 (1), 39-50.

Gil-Saura, I., Seric, M., Ruiz-Molina, M.E., \& Berenger-Contrí, G. (2016). The causal relationship between store equity and loyalty: testing two alternative models in retailing. Journal of Brand Management, 24 (2), 193-208. 
Gil-Saura, I., Ruiz-Molina, M.E., \& Berenger-Contrí, G. (2016). Store equity and behavioral intentions: the moderating role of the retailer's technology. Journal of Product \& Brand Management, 25 (7), 642-650.

Giraldi, J. M. E., Spinelli, P. B., \& Merlo, E. M. (2003). Retail store image: analysis of the implications for store positioning. Revista eletrônica de Administração, 9 (6), 207-208.

Hair Jr., J. F., Anderson, R. E., Tatham, R. L., \& Black, W. C. (2009). Multivariate Data Analysis. Upper Saddle River: Pearson Education.

Hartman, K. B., \& Spiro, R. L. (2005). Recapturing store image in costumer-based Store Equity: a construct conceptualizations. Journal of Business Research, 58, 1112-1120.

Iglesias, O., Markovic, S., Singh, J.J., \& Sierra, V. (2019). Do Customer Perceptions of Corporate Services Brand Ethicality Improve Brand Equity? Considering the Roles of Brand Heritage, Brand Image, and Recognition Benefits. Journal of Business Ethics, 154 (2), 441 459.

Keller, K. L. (2001). Building Customer-Based Brand Equity: A Blueprint for Creating Strong Brands, Marketing Science Institute, 01-107.

Keller, K. L., \& Lehmann, D. R. (2006). Brand and Branding: Research Findings and Future Priorities. Marketing Science, 25 (6), 740-759.

Kline, R. B. (1998). Principles and Practice of Structural Equation Modeling. New York: Guilford Press.

Larán, J. A., \& Espinoza, F. D. S. (2004). Consumidores satisfeitos, e então? Analisando a satisfação como antecedente da lealdade. Revista de Administração Contemporânea, 8 (2), 51-70.

Mangold, W. G., \& Faulds, D. (1993). Service Quality in a retail channel. The journal of services marketing, 7 (4), 4-11.

Martenson, R. (2007). Corporate brand image, satisfaction and store loyalty: A study of the store as a brand, store brands and manufacturer brands. International Journal of Retail \& Distribution Management, 35 (7), 544-555.

Martineau, P. (1958). The personality of the retail store. Harvard Business Review, 36 (1), 4755.

Miranda, F. D. (2009). Verificação da aplicabilidade do modelo store equity: um estudo em loja de material de construção, reformas e decoração da região metropolitana de Belo Horizonte. Dissertação de Mestrado, Pontifícia Universidade Católica de Minas Gerais, Belo Horizonte, MG, Brasil.

Munhoz, E. C. (2009). Aplicação do Modelo Store Equity: um estudo em um supermercado de Belo Horizonte. Dissertação de Mestrado, Pontifícia Universidade Católica de Minas Gerais, Belo Horizonte, MG, Brasil.

Muniz, E. C. V. (2009). Aplicação do Modelo Store Equity: um estudo em um supermercado de Belo Horizonte. Dissertação de Mestrado, Pontifícia Universidade Católica de Minas Gerais, Belo Horizonte, MG, Brasil.

Nikhashemi, S.R.; Tarofder, A.K., Gaur, S.S., \& Haque,A. (2016). The Effect of Customers' Perceived Value of Retail Store on Relationship between Store Attribute and Customer Brand Loyalty: Some Insights from Malaysia. Procedia Economics and Finances, 37, 432438.

Oliveira, A. C. (2010). Limites e Possibilidades de aplicação do modelo store equity: um estudo em uma panificadora na cidade de Campinas-SP. Dissertação de Mestrado, Pontifícia Universidade Católica de Minas Gerais, Belo Horizonte, MG, Brasil.

Oliveira, M. O. R., \& Luce, F. B. (2011). O valor da marca: conceitos, abordagens e estudos no Brasil. Revista Eletrônica de Administração, 17 (2), 502-529.

$\Theta$ Revista Gestão \& Tecnologia, Pedro Leopoldo, v. 19, n. 5, p. 101-121, out./dez. 2019 
Oliver, R. L. (1999). Whence consumer loyalty. Journal of Marketing, 63, 33-44.

Pappu, R., \& Quester, P. G. (2006). Does costumer satisfaction lead to improved Brand Equity? An empirical examination of two categories of retail brands. Journal of Product \& Brand Management, 15 (1), 4-14.

Rao, R. (2012). Brand Credibility and Brand Involvement as an Antecedent of Brand Equity: An Empirical Study. Asia-Pacific Journal of Business, 3 (1), 17-28.

Romaniuk, J., Sharp, B., Paech, S., \& Driesener, C. (2004). Brand and advertising awareness: a replication and extension of a known empirical generalization. Australian Marketing Journal, 12 (3), 70-80.

Rossoni, L., \& Guarido Filho, E. R. (2015). O que faz um nome? Status, conselho de administração e características organizacionais como antecedentes da reputação corporativa. Revista de Administração, 50 (3), 292-309.

Sasmita, J., \& Suki, N.M. (2015). Young consumers' insights on brand equity: Effects of brand association, brand loyalty, brand awareness, and brand image. International Journal of Retail \& Distribution Management, 43 (3), 276-292.

Sharma, P., Chen, I.S.N., \& Luk, S.T.K. (2018). Tourist Shopper's evaluation of retail service: a study of cross-border versus international outshoppers. Journal of Hospitality \& Tourism Research, 42 (3), 392-419.

Song, H.J., Wang, J.H., Han, H. (2019). Effect of image, satisfaction, trust, love, and respect on loyalty formation for name-brand coffee shops. International Journal of Hospitality Management, 79, 50-59.

Tabachnick, B. G., \& Fidell, L. S. (2007). Using Multivariate Statistics. Boston: Allyn and Bacon.

Teixeira, D. J., Oliveira, C. C. G., \& Pires, M. C. (2006). Store Equity: testando um modelo conceitual no varejo. In: Ângelo, C. F., \& Silveira, J. A. G (Orgs). Varejo Competitivo. São Paulo: Saint Paul Editora.

Teixeira, D. J., Oliveira, C. C. G., \& Pires, M. C. (2007). Store Equity: Testing a conceptual model in retail marketing. Anais do XIV International Conference on Recent Advances in Retailing and Services Science: The European Institute of Retailing and Services Studies (EIRASS), São Francisco, EUA.

Thang, D. C., \& Tan, B. L. B. (2003). Linking consumer perception to preference of retail stores: an empirical assessment of the multi-attributes of store image. Journal of Retailing and Consumer Services, 10 (4), 193-200.

Yoo, B., \& Donthu, N. (2001). Developing and validating a multidimensional customer-based Brand Equity scale. Journal of Business Research, 52 (1), 1-14. 